\title{
The production method affects the efficacy of platelet derivatives to expand mesenchymal stromal cells in vitro
}

\author{
Martina Bernardi 1,2, Francesco Agostini ${ }^{3}$, Katia Chieregato 1,2, Eliana Amati ${ }^{1}$, Cristina Durante ${ }^{3}$, Mario Rassu ${ }^{4}$, \\ Marco Ruggeri ${ }^{1}$, Sabrina Sella ${ }^{1}$, Elisabetta Lombardi ${ }^{3}$, Mario Mazzucato ${ }^{3 \dagger}$ and Giuseppe Astori ${ }^{{ }^{*}+}$ (D)
}

\begin{abstract}
Background: The use of fetal bovine serum (FBS) as a media supplement for the ex vivo expansion of bone-marrow derived mesenchymal stromal cells (BM-MSC) has been discouraged by regulatory agencies, due to the risk of transmitting zoonoses and to elicit immune reactions in the host once transplanted. Platelet derivatives are valid FBS substitutes due to their content of growth factors that can be released disrupting the platelets by physical methods or physiological stimuli. We compared platelet derivatives produced by freezing/thawing (platelet lysates, $\mathrm{PL}$ ) or after $\mathrm{CaCl}_{2}$ activation (platelet releasate surnatant rich in growth factors, PR-SRGF) for their content in growth factors and their ability to support the ex vivo expansion of BM-MSC.
\end{abstract}

Methods: The cytokine content in the two platelet derivatives was evaluated. BM-MSC were expanded in complete medium containing 10,7.5 and 5\% PL or PR-SRGF and the cell phenotype, clonogenic capacity, immunomodulation properties and tri-lineage differentiation potential of the expanded cells in both media were investigated.

Results: The concentration of PDGF-AB, PDGF-AA, PDGF-BB in PR-SRGF resulted to be respectively $5.7 \times, 1.7 \times$ and $2.3 \times$ higher compared to PL. PR-SRGF promoted a higher BM-MSC proliferation rate compared to PL not altering BM-MSC phenotype. Colony forming efficiency of BM-MSC expanded in PR-SRGF showed a frequency of colonies significantly higher than cells expanded in PL. BM-MSC expanded in PL or PR-SRGF maintained their immunomodulatory properties against activated lymphocytes even if BM-MSC expanded in FBS performed significantly better.

Conclusions: The method used to release platelet factors significantly affects the enrichment in growth factors and overall product performance. The standardization of the production process of platelet derivatives and the definition of their release criteria requires further investigation.

Keywords: Platelet lysate, Platelet releasate, Mesenchymal stromal cells, Mesenchymal stem cells, Ex vivo expansion, Fetal bovine serum

\section{Background}

Fetal bovine serum (FBS), rich in growth factors able to stimulate the cell proliferation is historically used for culturing cells [1, 2]. In 2008, the Committee for Medicinal Products for Human use of the European Medicine Agency discouraged the use of animal derivatives since

\footnotetext{
*Correspondence: astori@hemato.ven.it

${ }^{\dagger}$ Giuseppe Astori and Mario Mazzucato are senior authors

${ }^{1}$ Advanced Cellular Therapy Laboratory, Hematology Unit, Vicenza Hospital, Vicenza, Italy

Full list of author information is available at the end of the article
}

they may harbor infectious agents and may possibly increase undesirable immunological responses in the recipient [3]. As a consequence, there is an urgent need to find a replacement for FBS for the production of safe and reproducible cell therapy products.

The use of platelet lysate (PL) for the ex vivo expansion of mesenchymal stromal cells (MSC) was originally proposed by Doucet et al. [4]. There are strong evidences that PLs are able to support the expansion of bone marrow (BM) [5, 6], umbilical cord blood $[7,8]$ and adipose tissue derived-MSC [9]. 
Release of growth factors from platelets can be obtained through physical methods by freezing-thawing the platelet rich plasma obtained from the platelet apheresis or from the buffy coat. Briefly, the platelet rich plasma bags are frozen overnight at $-80{ }^{\circ} \mathrm{C}$ and then thawed at $+37{ }^{\circ} \mathrm{C}$; the cycle is repeated for $1-3$ times. After pooling and one or more centrifugation/filtration steps in order to remove cellular debris, PL is ready for being added to the growth media [10].

Alternatively, platelets can be disrupted by sonication as formerly described by Hara et al. [11] and by our group [12].

Platelet factors can be also released by physiological stimuli as thrombin, collagen, adenosine diphosphate, epinephrine and thrombin receptor-activating peptide, $\mathrm{CaCl}_{2}$ or tri-n-buthyl phosphate in order to obtain the socalled platelet releasate (PR) $[13,14]$. The method used to release the platelet factors could represent a variable able to influence the quality of the final product but only few studies have addressed this issue so far.

In this work we have produced PL by freezing/thawing and platelet releasate by $\mathrm{CaCl}_{2}$ activation in order to obtain the so-called platelet releasate surnatant rich in growth factors PR-SRGF [15]. The two platelet derivatives were compared for their content in selected growth factors. Moreover, BM-MSC were expanded in complete medium containing 10, 7.5 and 5\% PL or PR-SRGF and the cell phenotype, clonogenic capacity, immunomodulation properties and tri-lineage differentiation potential of the expanded cells in both media were investigated.

\section{Methods}

\section{Preparation of PL}

Platelet apheresis were collected from 15 donors by using a Trima Accel separator (Caridian BCT Inc, Lakewood, CO, USA), transferred to 50-ml tubes (Falcon, Corning MA, USA) and stored at $-80^{\circ} \mathrm{C}$. After 2 cycles of freezing/thawing the aliquots were centrifuged at $1600 \times g$ for $15 \mathrm{~min}$ at room temperature and the supernatants were collected, pooled, filtered using a $70 \mu \mathrm{m}$ cell strainer (Falcon, Corning MA, USA) and finally stored at $-20{ }^{\circ} \mathrm{C}$ until use.

\section{Preparation of PR-SRGF}

Platelet apheresis were collected from 15 donors by using a multicomponent collection system (Haemonetics, MA, USA). Platelet activation was performed by adding $\mathrm{CaCl}_{2}$ (Monico, Venice, Italy) at the final concentration of $0.04 \mathrm{M}$ and after incubation at $40{ }^{\circ} \mathrm{C}$ for approximately 60 min until complete clot formation. Bags were centrifuged for $5 \mathrm{~min}$ at $2200 \times g$ and the SRGF collected and stored at $-80^{\circ} \mathrm{C}$.

\section{Cytokine determination}

Platelet-derived growth factor (PDGF)-AA, PDGF$A B, P D G F-B B$, epidermal growth factor (EGF), vascular endothelial growth factor (VEGF), basic fibroblast growth factor (FGF-basic), insulin like growth factor-1 (IGF-1) and transforming growth factor- $\beta 1$ (TGF- $\beta 1$ ) were quantified by using a Quantikine ELISA Kits (R\&D Systems, Minneapolis, MN, USA).

\section{BM-MSC isolation and expansion}

Total nucleated cells were isolated from the washout and filters used for bone marrow collection $(\mathrm{n}=3)$. Cells were counted and plated at 100,000 cells $/ \mathrm{cm}^{2}$ in low glucose Dulbecco's modified Eagle's medium + glutamax (Gibco Invitrogen, Carlsbad, CA, USA) containing penicillin/streptomycin (Sigma-Aldrich, Saint Louis, Missouri, USA) and heparin $30 \mathrm{U} / \mathrm{ml}$ (Hospira Italia, Napoli, I) supplemented with 10\% FBS (Gibco Invitrogen, Carlsbad, CA, USA) or $7.5 \%$ platelet derivatives. Cells were incubated at $37{ }^{\circ} \mathrm{C}$ and $5 \% \mathrm{CO}_{2}$ concentration. After 3-4 days in culture, non-adherent cells were removed and fresh medium was added (P0). The resulting plastic adherent cells were expanded until $80-90 \%$ confluence and then harvested with TrypLE ${ }^{\mathrm{TM}}$ Select (Gibco Invitrogen, Carlsbad, CA, USA) and counted (P0).

BM-MSC were thawed and expanded in presence of $10 \%$ FBS or $5,7.5$ and $10 \%$ PL or PR-SRGF. At each subcultivation, the population doubling (PD) was calculated as follows: $\mathrm{PD}=\log 10(\mathrm{~N}) / \log 10(2)$; where $\mathrm{N}$ is the number of cells harvested-the number of cells initially seeded. The cumulative PD (cPD) was calculated adding to the PD of the passage under analysis the PDs of the previous passages.

\section{Immunophenotypic analysis}

Cells were stained at P4 and P7 following the International Society for Cellular Therapy guidelines [16] with anti-human antibodies against CD31, CD34, CD45, CD105, CD44, CD90 (all from Beckman Coulter, Fullerton, CA, USA) and CD73, (Beckton Dickinson, Franklin Lakes, NJ, USA). Briefly, about $1 \times 10^{5}$ cells were incubated for $15 \mathrm{~min}$ at room temperature with the specific monoclonal antibody. At least 10,000 events were acquired by using a CYTOMICS FC500 flow cytometer (Beckman Coulter Fullerton, CA, USA).

\section{Immunomodulation}

Immunomodulation analysis was performed by co-culturing BM-MSC with peripheral blood mononucleated cells (PBMC) isolated by density gradient centrifugation and labeled with Carboxyfluorescein Succinimidyl ester (Celltrace CFSE Cell Proliferation Kit, Invitrogen, Carlsbad, CA, USA) at the PBMC:BM-MSC ratio of 20:1, 
10:1 and 5:1. PBMC were stimulated with $500 \mathrm{U} / \mathrm{ml}$ of Interleukin 2 (Proleukin, Novartis Pharma, Varese, I) and $0.5 \mu \mathrm{g} / \mathrm{ml}$ antibody anti CD3 (Miltenyi Biotec, Bergisch Gladbach, DE). PBMC unstimulated and activated in absence of effector cells were used as controls. After 6 days, PBMC were labeled with CD45 PC7 (Beckman Coulter Fullerton, CA, USA) and 7-aminoactinomycin D (7-AAD) (Invitrogen Carlsbad, CA, USA) and analyzed with a FC500 flow cytometer (Beckman Coulter Fullerton, CA, USA). The inhibition of the PBMC CD45+ 7AAD-cell subset induced by BM-MSC was expressed according to the following formula:

\section{Colony forming efficiency}

At passage 2 (P2) and 5 (P5), 200 BM-MSC were plated in duplicate in 100-mm diameter culture dishes (Cellstar, Grainer Bio-One GmbH, Germany) in medium containing $10 \%$ FBS, $7.5 \% \mathrm{PL}$ and $7.5 \%$ PR-SRGF respectively. The cell medium were changed weekly and after 14 days the cells were fixed with $10 \%$ formalin and stained with May-Grunwald Giemsa. Stained plates were scored for colony forming units (CFU) by an inverted light microscope (Axiovert 40 CFL, Zeiss, Germany). Colonies consisting of at least 30 cells were counted as CFU.

$\left(\% \mathrm{CFSE}^{+}\right.$activated PBMC in absence of BM-MSC -

$\% \mathrm{CFSE}^{+}$activated PBMC in presence of BM-MSC) $\times 100$ $\% \mathrm{CFSE}^{+}$activated PBMC in absence of BM-MSC

\section{BM-MSC tri-lineage differentiation}

For osteogenic and adipogenic differentiation, BM-MSC at the end of passage 4 were seeded at a density of 4000 cells/ $\mathrm{cm}^{2}$ on cell culture coverslips (Thermo Fisher Scientific, Waltham, MA, USA) arranged in 24-well plates (Falcon, Corning, NY, USA) in presence of medium supplemented with $10 \%$ FBS, $7.5 \%$ PL or $7.5 \%$ PR-SRGF. At $70-80 \%$ of cell confluence, the medium was replaced with specific differentiation media, then renewed every 3-4 days for 21 days. To induce adipogenic differentiation, cells were incubated using the StemPro adipogenic differentiation kit (Thermo Fisher Scientific, Waltham, MA, USA), according to the manufacturer's instructions. The presence of intracellular lipid droplets was detected by standard staining with Oil Red O (Diapath, Bergamo, Italy), according to the manufacturer's instructions. In parallel, cells were also grown using the StemPro ${ }^{\circledR}$ osteogenic differentiation kit (Thermo Fisher Scientific, Waltham, MA, USA) to induce osteogenic differentiation. The presence of calcium deposit was evaluated by von Kossa staining (Sigma-Aldrich, Saint Louis, MO, USA). Cells were fixed with $10 \%$ formalin for $5 \mathrm{~min}$ at room temperature, incubated with $1 \%$ silver nitrate solution for $15 \mathrm{~min}$ and exposed to ultraviolet light for $2 \mathrm{~h}$. Coverslips were rinsed with distilled water and 5\% sodium thiosulfate to remove unreacted silver. Finally, cells were counterstained with Nuclear Fast Red Solution (Sigma-Aldrich, Saint Louis, MO, USA). To induce chondrogenesis, $10 \times 10^{4}$ cells in $100 \mu \mathrm{l}$ chondrogenic induction medium (StemPro chondrogenic differentiation kit, Thermo Fisher Scientific, Waltham, MA, USA) were placed in a 24-well plates (Falcon, Corning, NY, USA) and after an incubation at $37{ }^{\circ} \mathrm{C}$ for about $12 \mathrm{~h}, 700 \mu \mathrm{l}$ chondrogenic induction medium were added. Fresh chondrogenic medium was added every 3-4 days. After 28 days, the micromass was fixed with formalin 10\% and stained with Alcian Blue 1\% acetic acid (Sigma-Aldrich, Saint Louis, MO, USA).

\section{Statistical analysis}

The analysis of variance was calculated by using the two way ANOVA test by using the GraphPad Prism software (GraphPad Prism Software version 5.01, Inc. La Jolla, CA, USA).

\section{Results \\ Cell proliferation}

PR-SRGF promoted a higher proliferation rate compared to PL and FBS resulting in a greater cPD until P8 at any supplement concentration. In particular, at P5 the cPD of BM-MSC expanded in presence of PR-SRGF was significantly higher compared to PL at each concentration ( $\mathrm{P}<0.05$ for the $5 \%$ and $\mathrm{P}<0.01$ for the 7.5 and $10 \%$ conditions). Complete data are reported in Fig. 1.

\section{Quantification of platelet factors released}

PL and PR-SRGF were produced from two different pools of 15 donors each. The final volume of PL and PR-SRGF obtained was comparable. The concentration of PDGF$A B$, PDGF-AA, PDGF-BB in PR-SRGF resulted to be respectively $5.7 \times, 1.7 \times$ and $2.3 \times$ higher when compared with PL. The concentrations of VEGF, EGF and TGF- $\beta 1$ were comparable. Interestingly, IGF-1 was not detected in PL (Table 1).

\section{Immunophenotype}

Expanded BM-MSC were strongly positive ( $\geq 95 \%)$ for CD90, CD44, CD105 and CD73 at P4 and P7 in presence of FBS, PL or PR-SRGF. CD31 and hematopoietic cell markers CD34 and CD45 were not expressed evidencing the isolation of homogeneous cell population.

\section{Immunomodulation}

BM-MSC expanded in 10\% FBS, PL or PR-SRGF maintained their ability to inhibit proliferation of activated lymphocytes. The percentage of inhibition was directly 

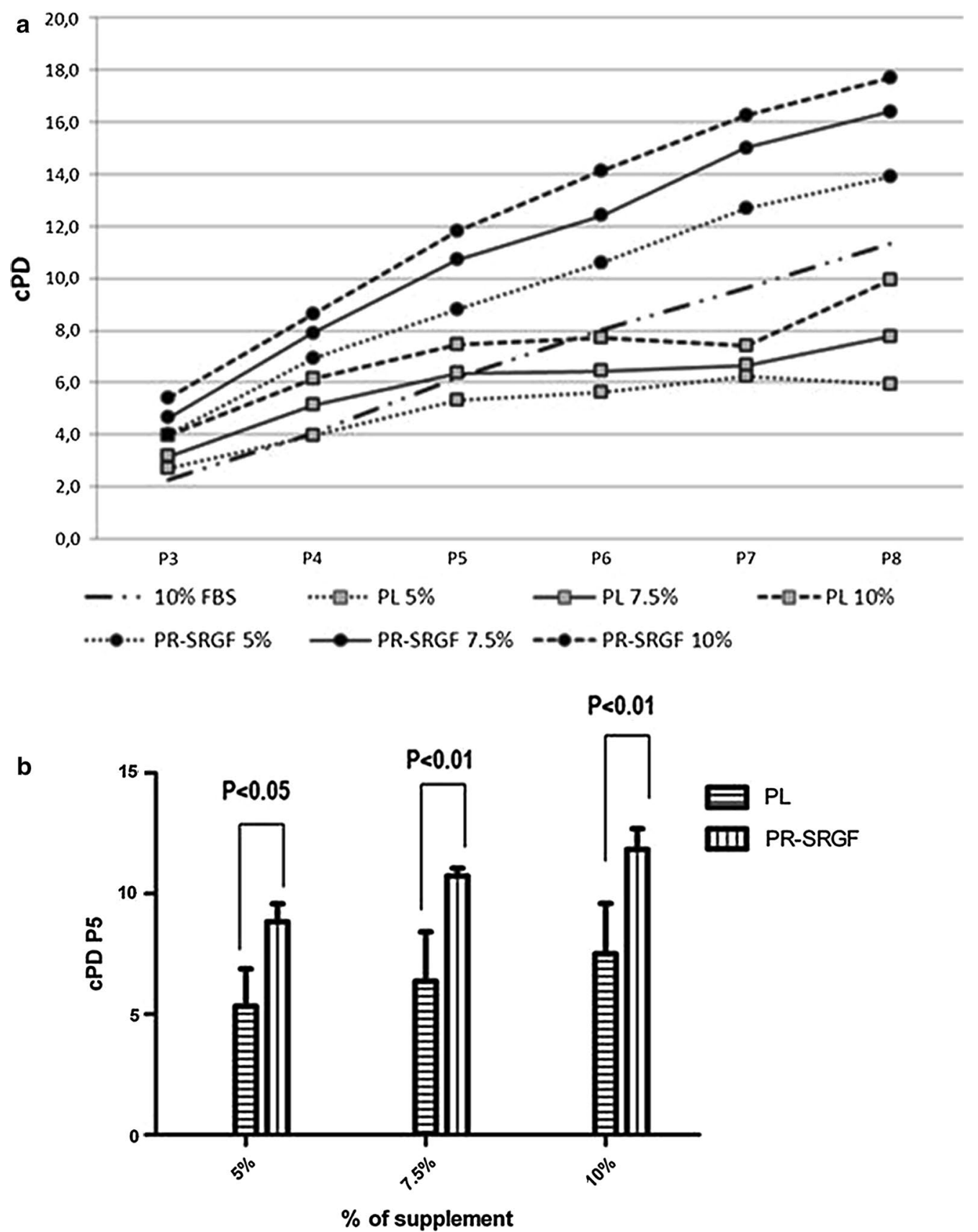

Fig. 1 CPD of BM-MSC expanded in presence of PL, PR-SRGF or FBS. a CPD values of BM-MSC cultured at different \% of supplement from P3 to P8. PR-SRGF promotes a higher proliferation rate compared to PL and FBS resulting in a greater CPD until P8 at any supplement concentration. $\mathbf{b}$ Comparison of CPD at P5 between BM-MSC expanded in presence of PR-SRGF or PL at different \% of supplement. BM-MSC expanded in presence of PR-SRGF showed a higher CPD than those expanded in PL. Statistical analysis was performed by two-way ANOVA $(n=3)$

proportional with the number of BM-MSC seeded. Immunomodulation of BM-MSCs expanded in PL and PR-SRGF was comparable but significantly lower when compared with FBS. In detail, BM-MSC expanded in FBS showed a significant difference for the ratio 20:1 versus BM-MSC expanded in PL $(\mathrm{P}<0.01)$ and a significant difference for all the ratios versus BM-MSC expanded in PR-SRGF $(\mathrm{P}<0.05)$ (Fig. 2). 
Table 1 Concentration of growth factors in PL, PR-SRGF and related fold increase

\begin{tabular}{llll}
\hline Growth factor & PL & PR-SRGF & PR-SRGF/PL fold increase \\
\hline VEGF, pg/ml & $0.56 \pm 0.15 \times 10^{3}$ & $0.52 \pm 0.03 \times 10^{3}$ & $0.9 \times$ \\
EGF, pg/ml & $1.65 \pm 0.49 \times 10^{3}$ & $1.95 \pm 0.16 \times 10^{3}$ & $1.2 \times$ \\
PDGF-AB, pg/ml & $25.16 \pm 6.36 \times 10^{3}$ & $142.66 \pm 25.25 \times 10^{3}$ & $5.7 \times$ \\
PDGF-AA, pg/ml & $4.78 \pm 0.35 \times 10^{3}$ & $8.30 \pm 2.23 \times 10^{3}$ & $1.7 \times$ \\
PDGF-BB, pg/ml & $5.06 \pm 1.24 \times 10^{3}$ & $11.53 \pm 1.58 \times 10^{3}$ & $2.3 \times$ \\
TGF- $\beta 1, \mathrm{pg} / \mathrm{ml}$ & $53.04 \pm 12.07 \times 10^{3}$ & $39.80 \pm 10.10 \times 10^{3}$ & $0.8 \times$ \\
IGF- $1, \mathrm{ng} / \mathrm{ml}$ & 0.00 & $0.11 \pm 0.006 \times 10^{3}$ & - \\
FGF-basic, pg/ml & $0.085 \pm 0.008 \times 10^{3}$ & $0.011 \pm 0.004 \times 10^{3}$ & $0.1 \times$ \\
\hline
\end{tabular}

Data are expressed as mean $\pm S D$

\section{Tri-lineage differentiation}

BM-MSC expanded at P5 in presence of 10\% FBS, 7.5\% PL and 7.5\% PR-SRGF were able to maintain their tri-lineage differentiation potential (Fig. 3).

\section{Colony forming efficiency}

The colony forming efficiency of BM-MSC expanded in presence of PR-SRGF was significant higher compared to BM-MSC expanded in PL at P2 $(\mathrm{P}<0.01)$ and P5 $(\mathrm{P}<0.05)$. To note that BM-MSC expanded in presence of FBS showed a significantly higher capacity to produce colonies than the BM-MSC expanded in presence of PL and PR-SRGF at P2 and P5 (P < 0.001) (Fig. 4).

\section{Discussion}

The freezing/thawing procedure is widely used to release growth factors from platelets whereas methods based on physiological activation requiring the addition of specific compounds to the platelet concentrate are not commonly applied. Nevertheless, the impact that the method used to release platelet factors could have on the composition of the final product and on the cell expansion performance has not fully investigated. It has been observed that PR obtained after platelet activation with thrombin significantly accelerated BM-MSC proliferation compared to PL obtained by freezing/thawing even if cell surface marker expression, adipogenic and osteogenic differentiation, and immunosuppressive action were similar in MSC from all culture conditions [5].

Previous works also reported that repeated freezing/ thawing cycles could have negative effects on the integrity of platelet growth factors $[17,18]$. Moreover, the addition of thrombin or $\mathrm{CaCl}_{2}$ depletes coagulation factors like fibrinogen or von Willebrand factor and adhesive proteins like fibronectin possibly influencing the composition of the final product. All these variables could result in functional differences between PL and PR.

To clarify if the method used to release growth factors could influence the cell phenotype, the ability to support the ex vivo expansion and the immunomodulatory properties of BM-MSC we have expanded the BM-MSC in presence of PL and PR-SRGF starting from a pool of 15 samples in order to reduce the between-sample variability.

We have compared the two supplements for their content in selected growth factors. It was demonstrated that PDGF and TGF- $\beta$ families of cytokines are able to affect cell morphogenesis, proliferation and differentiation once released from platelets [19]. In particular, PDGF-AB/BB, TGF- $\beta 1$ and FGF-basic are essential stimuli for the proliferation of MSC even if on their own these three factors are insufficient to promote MSC expansion. Therefore, other constituents of PL/PR should be present in the final product acting in synergy. In our experience, the content in growth factors resulted to be different in PL or PRSRGF. Taking as arbitrarily relevant a fold increase $\geq 1.5 \times$ the concentration of PDGF-AB, PDGF-AA, PDGF-BB in PR-SRGF resulted to be higher $(5.7 \times, 1.7 \times$ and $2.3 \times$, respectively) compared to the concentrations found in PL. Nevertheless to understand if the observed differences could be biologically related to the expansion rate changes, further investigations are needed.

Focusing the analysis of cPD at passage 5 (this is the accepted limit for harvesting MSC cells for clinical use) we have observed that BM-MSC expanded more rapidly in $5,7.5,10 \%$ PR-SRGF than in PL (Fig. 1).

The immunophenotype of the cells did not show any difference of expression on the cells expanded in the three different media suggesting that at least for the surface markers that we have analyzed the phenotype was not influenced by the addition of PL or PR-SRGF and fitted with ISCT criteria regardless of the analyzed supplements at each concentration.

MSC exert potent immunosuppressive and antiinflammatory activities [20] suppressing $\mathrm{T}$ cell proliferation in vitro by the production of soluble factors, including indoleamine 2,3-dioxygenase [21]. We evidenced that BM-MSC expanded in $7.5 \%$ PL or $7.5 \%$ 

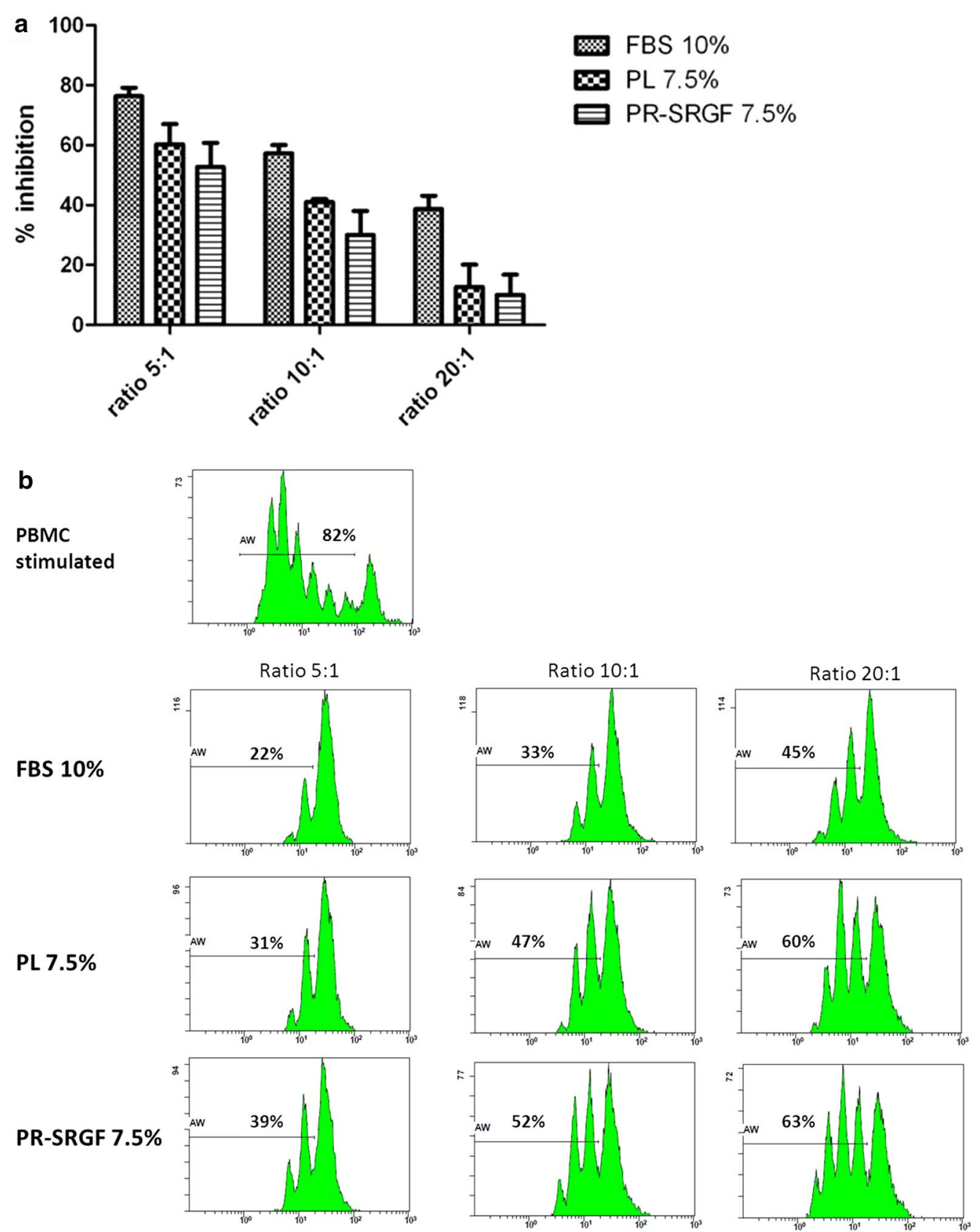

Fig. 2 Immunomodulation properties of BM-MSC expanded in different supplements. a Inhibitory effect BM-MSC on CFSE labeled PBMC. Cells were co-cultured at three different PBMC:BM-MSC ratios upon PBMC stimulation. Each bar represents mean \pm SD of \% inhibition of three independent experiments. Immunomodulation of BM-MSCs expanded in PL and PR-SRGF was comparable but significantly lower when compared with FBS. Three BM-MSC batches were analyzed by two-way ANOVA. b PBMC proliferation was assessed by CFSE dilution method on CD45+ cells. One representative case is shown

PR-SRGF maintained a comparable ability to inhibit the lymphocyte proliferation and the percentage of inhibition was directly proportional with the number of BMMSC seeded. It should be noted that BM-MSC expanded in $10 \%$ FBS maintained better immunomodulatory properties. This is still a matter of debate. In-fact, some authors have observed that MSC expanded in presence of PL retained their immunosuppressive capabilities [5, 22] whereas others suggested that PL-expanded MSC showed reduced immunosuppressive potential [23, 24]. 


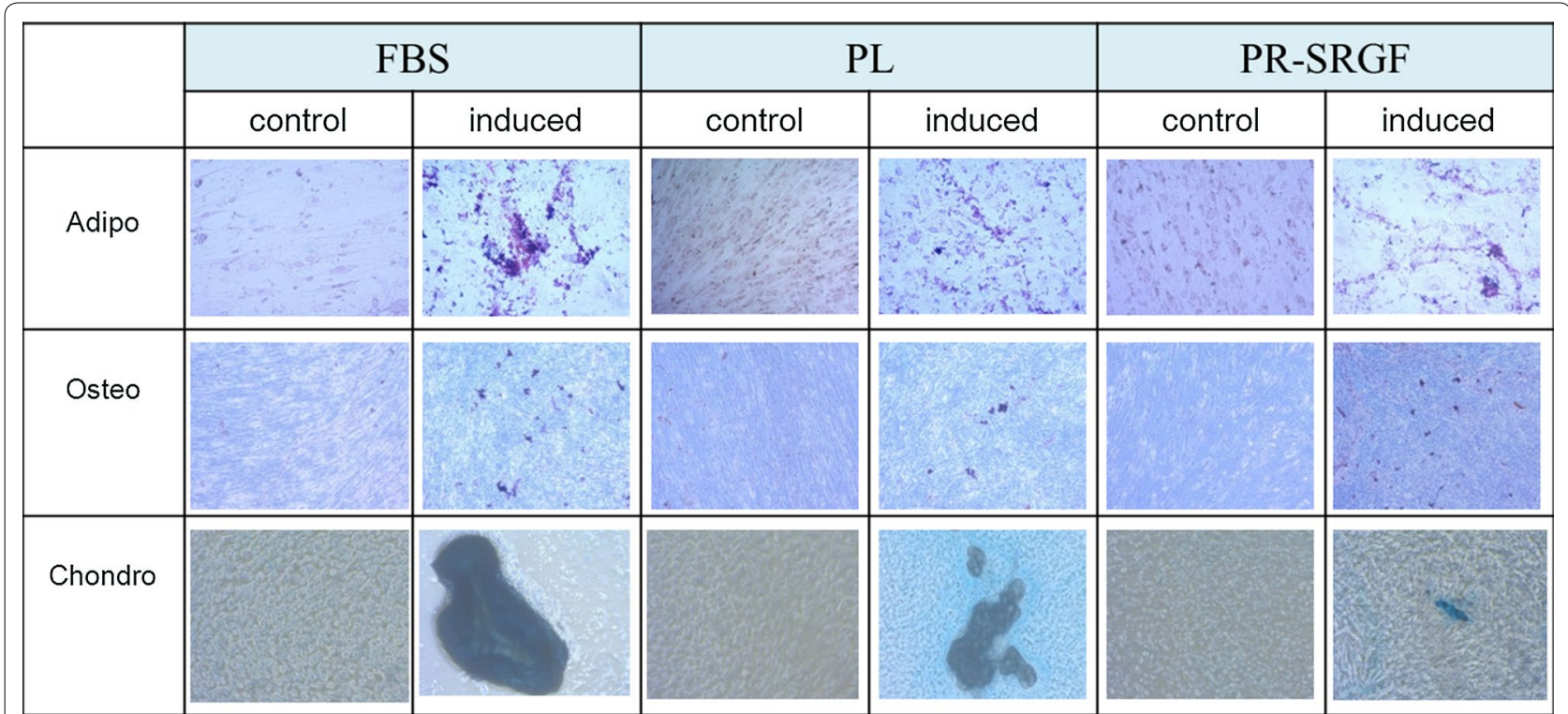

Fig. 3 Tri-lineage differentiation. Differentiation potential into adipogenic, osteogenic and chondrogenic lineages was performed on BM-MSC cultured in presence of FBS, PL and PR-SRGF. One representative assay is reported
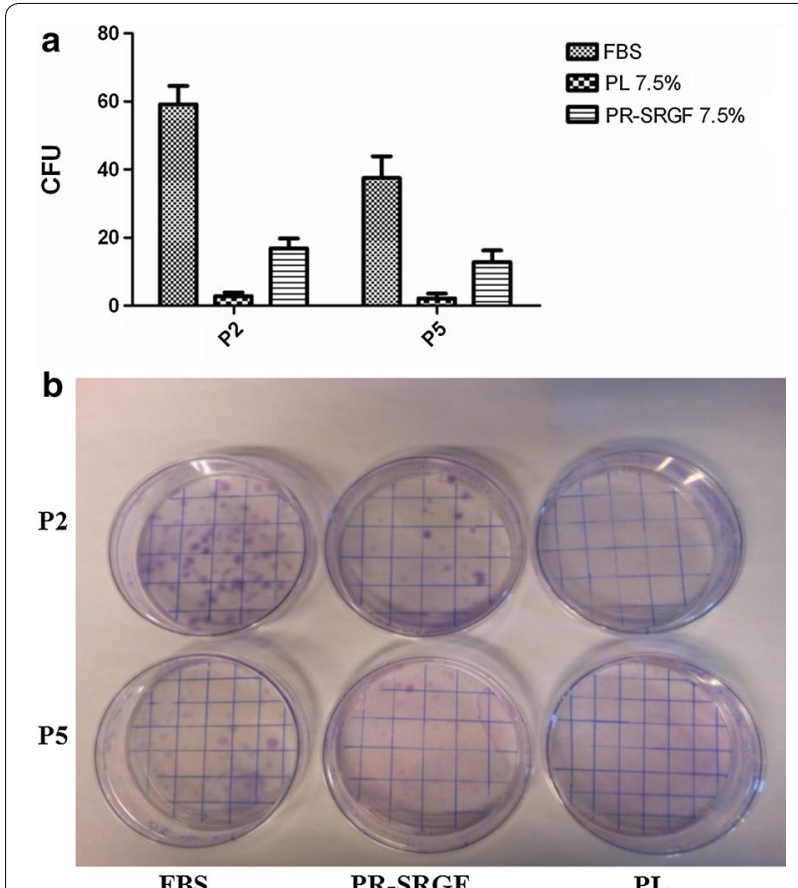

Fig. 4 Colony forming efficiency of BM-MSC expanded in presence of the three supplements. a Each bar represents mean \pm SD of CFU of three independent experiments. BM-MSC expanded in presence of FBS showed a significantly higher capacity to produce colonies than BM-MSC expanded in presence of PL and PR-SRGF at P2 and P5 $(P<0.001)$. Three BM-MSC batches were analyzed by two-way ANOVA test. b Colonies formed after plating 200 MSC in 100-mm culture dishes are shown from one representative case at P2 and P5
Discrepancies about immunomodulation results between different research groups could reflect the different PL manufacturing process and further studies are required to clarify the matter.

We did not have observed a direct correlation between CPD and the colony forming efficiency of BM-MSC expanded in presence of FBS or platelet derivatives. Infact, at P2 and P5 the CFU of BM-MSC expanded in presence of FBS was significantly better when compared with BM-MSC expanded in presence of PL and PR-SRGF.

Our hypothesis is that FBS, maybe due to its different composition in growth factors is able to better support the colony formation due to an improved capacity to allow the attachment of the MSC clones to the plastic surface of the petri dish whereas platelet derivatives have a higher proliferative capacity leading to an increased cell proliferation able to produce a large amount of cells in the following passages as confirmed by cPD values.

\section{Conclusions}

We demonstrated that the method used to release platelet factors influence the composition of the product in terms of growth factor concentration and product performance. PR-SRGF obtained by platelet activation with $\mathrm{CaCl}_{2}$ promotes BM-MSC proliferation significantly better than PL not altering the cell phenotype and maintaining trilineage differentiation capacity of the expanded cells. Also the clonogenic capacity of BM-MSC expanded in PR-SRGF is better than PL. It is mandatory to understand 
the mechanisms that mediate the beneficial effects of platelet factors on cell growth with the aim to standardize the production process and to define adequate release criteria. This requires an effort between all the stakeholders involved in the manufacturing and use of this product.

\begin{abstract}
Abbreviations
PL: platelet lysate; PR-SRGF: platelet releasate surnatant rich in growth factors; BM-MSC: bone-marrow derived mesenchymal stromal cells; FBS: fetal bovine serum; PDGF: platelet-derived growth factor; EGF: epidermal growth factor; VEGF: vascular endothelial growth factor; FGF-basic: basic fibroblast growth factor; IGF-1: insulin like growth factor-1; TGF- $\beta$ : transforming growth factor- $\beta 1$; PD: population doubling; CPD: cumulative population doubling; CFSE: carboxyfluorescein succinimidyl ester; PBMC: peripheral blood mononucleated cells; 7-AAD: 7-aminoactinomycin D.
\end{abstract}

\section{Authors' contributions}

$M B, F A, E L$ and KC participated in the study concept and designed, performed experiments and analyzed data. MB and GA wrote the manuscript. GA, MM and FA critically revised the article. All authors read and approved the final manuscript.

\section{Author details}

1 Advanced Cellular Therapy Laboratory, Hematology Unit, Vicenza Hospital, Vicenza, Italy. ${ }^{2}$ Hematology Project Foundation, Contrà S. Francesco 41, Vicenza, Italy. ${ }^{3}$ Stem Cell Collection and Processing Unit, CRO National Cancer Institute-IRCCS Aviano, Aviano, Italy. ${ }^{4}$ Department of Microbiology, San Bortolo Hospital, Via Rodolfi 37, 36100 Vicenza, Italy.

\section{Acknowledgements}

Thanks to the donors for supplying and to the physicians and nurses of the Hematology of the Vicenza Hospital for collecting the bone marrow. Thanks also to technicians and nurses of the Cell Collection and Processing Unit of the CRO Aviano National Cancer Institute-IRCCS.

\section{Competing interests}

The authors declare that they have no competing interests.

\section{Availability of data and materials}

All data generated or analyzed during this study are available from the corresponding author on reasonable request.

\section{Ethics approval and consent to participate}

The ethics approval was released by the "Comitato Etico per le Sperimentazioni Cliniche della Provincia di Vicenza", act 4/15F, on January, 19th, 2015. The authors state that they have followed the principles outlined in the Declaration of Helsinki for all investigations. Informed written consent has been obtained from the individuals whose samples were involved in experimental investigations

\section{Funding}

This work was supported in part by grants obtained by M.M. from Ministero della Salute, Rome, Italy (Grant Code RF-2010-2317993) and by Associazione Italiana contro Linfomi, Mielomi e Leucemie (AIL); by grants obtained by Mauro Krampera, University of Verona (Italy) from Fondazione Cariverona (Research Project 2012.0828) and from Ricerca Sanitaria Finalizzata Regionale del Veneto (Research Project 334/12) and also by grants assigned to GA obtained from Associazione Vicentina per le Leucemie ed i Linfomi-AlL and Associazione Donatori di Sangue Cavaliere Pietro Trevisan, Montecchio Maggiore.

\section{Publisher's Note}

Springer Nature remains neutral with regard to jurisdictional claims in published maps and institutional affiliations.

Received: 16 September 2016 Accepted: 14 April 2017

Published online: 01 May 2017
References

1. Witkowski JA. Alexis Carrel and the mysticism of tissue culture. Med Hist. 1979;23(3):279-96

2. Brindley DA, Davie NL, Culme-Seymour EJ, Mason C, Smith DW, Rowley JA. Peak serum: implications of serum supply for cell therapy manufacturing. Regen Med. 2012;7(1):7-13.

3. Guideline on Human Cell-Based Medicinal Products. European Medicine Agency. Committee for Medicinal Product for Human use. 2008.

4. Doucet C, Ernou I, Zhang Y, Llense JR, Begot L, Holy X, Lataillade JJ. Platelet lysates promote mesenchymal stem cell expansion: a safety substitute for animal serum in cell-based therapy applications. J Cell Physiol. 2005;205(2):228-36.

5. Bieback K, Hecker A, Kocaomer A, Lannert H, Schallmoser K, Strunk D, Kluter H. Human alternatives to fetal bovine serum for the expansion of mesenchymal stromal cells from bone marrow. Stem Cells. 2009;27(9):2331-41.

6. Bartmann C, Rohde E, Schallmoser K, Purstner P, Lanzer G, Linkesch W, Strunk D. Two steps to functional mesenchymal stromal cells for clinical application. Transfusion. 2007;47(8):1426-35.

7. Avanzini MA, Bernardo ME, Cometa AM, Perotti C, Zaffaroni N, Novara F, Visai L, Moretta A, Del Fante C, Villa R, et al. Generation of mesenchymal stromal cells in the presence of platelet lysate: a phenotypic and functional comparison of umbilical cord blood-and bone marrow-derived progenitors. Haematologica. 2009;94(12):1649-60.

8. Reinisch A, Bartmann C, Rohde E, Schallmoser K, Bjelic-Radisic V, Lanzer G, Linkesch W, Strunk D. Humanized system to propagate cord bloodderived multipotent mesenchymal stromal cells for clinical application. Regen Med. 2007;2(4):371-82.

9. Blande IS, Bassaneze V, Lavini-Ramos C, Fae KC, Kalil J, Miyakawa AA, Schettert IT, Krieger JE. Adipose tissue mesenchymal stem cell expansion in animal serum-free medium supplemented with autologous human platelet lysate. Transfusion. 2009:49(12):2680-5.

10. Schallmoser K, Strunk D. Preparation of pooled human platelet lysate $(\mathrm{pHPL})$ as an efficient supplement for animal serum-free human stem cell cultures. J Vis Exp. 2009;(32):e1523.

11. Hara Y, Steiner M, Baldini MG. Platelets as a source of growth-promoting factor(s) for tumor cells. Cancer Res. 1980;40(4):1212-6.

12. Bernardi M, Albiero E, Alghisi A, Chieregato K, Lievore C, Madeo D, Rodeghiero F, Astori G. Production of human platelet lysate by use of ultrasound for ex vivo expansion of human bone marrow-derived mesenchymal stromal cells. Cytotherapy. 2013;15(8):920-9.

13. Astori G, Amati E, Bambi F, Bernardi M, Chieregato K, Schafer R, Sella S, Rodeghiero F. Platelet lysate as a substitute for animal serum for the ex vivo expansion of mesenchymal stem/stromal cells: present and future. Stem Cell Res Ther. 2016;7(1):93.

14. Bieback K. Platelet lysate as replacement for fetal bovine serum in mesenchymal stromal cell cultures. Transfus Med Hemother. 2013;40(5):326-35.

15. Durante C, Agostini F, Abbruzzese L, Toffola RT, Zanolin S, Suine C, Mazzucato M. Growth factor release from platelet concentrates: analytic quantification and characterization for clinical applications. Vox Sang. 2013;105(2):129-36.

16. Dominici M, Le Blanc K, Mueller I, Slaper-Cortenbach I, Marini F, Krause D, Deans R, Keating A, Prockop D, Horwitz E. Minimal criteria for defining multipotent mesenchymal stromal cells. The International Society for Cellular Therapy position statement. Cytotherapy. 2006:8(4):315-7.

17. Mojica-Henshaw MP, Jacobson P, Morris J, Kelley L, Pierce J, Boyer M, Reems JA. Serum-converted platelet lysate can substitute for fetal bovine serum in human mesenchymal stromal cell cultures. Cytotherapy. 2013;15(12):1458-68.

18. Laitinen A, Oja S, Kilpinen L, Kaartinen T, Moller J, Laitinen S, Korhonen M, Nystedt J. A robust and reproducible animal serum-free culture method for clinical-grade bone marrow-derived mesenchymal stromal cells. Cytotechnology. 2016;68(4):891-906. doi:10.1007/s10616-014-9841-x.

19. Fekete N, Gadelorge M, Furst D, Maurer C, Dausend J, Fleury-Cappellesso S, Mailander V, Lotfi R, Ignatius A, Sensebe L, et al. Platelet lysate from whole blood-derived pooled platelet concentrates and apheresis-derived platelet concentrates for the isolation and expansion of human bone marrow mesenchymal stromal cells: production process, content and identification of active components. Cytotherapy. 2012;14(5):540-54.

20. Keating A. How do mesenchymal stromal cells suppress T cells? Cell Stem Cell. 2008;2(2):106-8. 
21. Meisel R, Zibert A, Laryea M, Gobel U, Daubener W, Dilloo D. Human bone marrow stromal cells inhibit allogeneic T-cell responses by indoleamine 2,3-dioxygenase-mediated tryptophan degradation. Blood. 2004;103(12):4619-21.

22. Flemming A, Schallmoser K, Strunk D, Stolk M, Volk HD, Seifert M. Immunomodulative efficacy of bone marrow-derived mesenchymal stem cells cultured in human platelet lysate. J Clin Immunol. 2011;31(6):1143-56.

23. Abdelrazik H, Spaggiari GM, Chiossone L, Moretta L. Mesenchymal stem cells expanded in human platelet lysate display a decreased inhibitory capacity on T- and NK-cell proliferation and function. Eur J Immunol. 2011;41(11):3281-90

24. Bernardo ME, Avanzini MA, Perotti C, Cometa AM, Moretta A, Lenta E, Del Fante C, Novara F, de Silvestri A, Amendola G, et al. Optimization of in vitro expansion of human multipotent mesenchymal stromal cells for cell-therapy approaches: further insights in the search for a fetal calf serum substitute. J Cell Physiol. 2007;211(1):121-30.

\section{Submit your next manuscript to BioMed Central and we will help you at every step:}

- We accept pre-submission inquiries

- Our selector tool helps you to find the most relevant journal

- We provide round the clock customer support

- Convenient online submission

- Thorough peer review

- Inclusion in PubMed and all major indexing services

- Maximum visibility for your research

Submit your manuscript at

www.biomedcentral.com/submit 